\title{
APPENDIX: Methodology
}

\section{From Silos to Systems: Investing in Sustainable Nutrition Science for a Healthy Future}

www.ucsusa.org/resources/from-silos-to-systems

https://doi.org/10.47923/2021.14270

\section{Table of Contents}

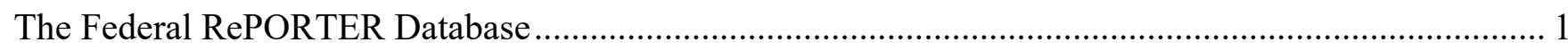

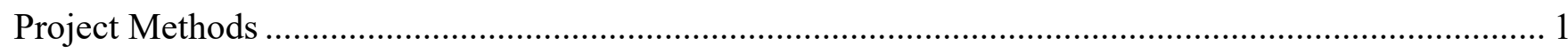

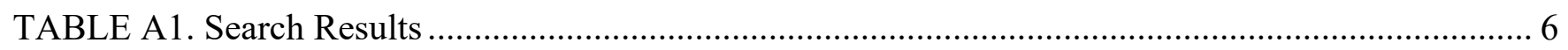

TABLE A2. Funding Estimates, by Year (Figure 2 data) ......................................................... 11

TABLE A3. Federal Recommendations for Sustainable Nutrition Science, 2010-2021 ..................... 12

FIGURE A1. Framework for Sustainable Nutrition Science.................................................... 14

FIGURE A2. PRISMA Flow Diagram for Project Search and Selection......................................... 15

\section{The Federal RePORTER Database}

UCS used the National Institutes of Health (NIH) Federal RePORTER database as the source for the From Silos to Systems analysis because it is the single publicly available database that links disparate data sources on scientific awards made by the federal government's 18 primary science agencies and transparently provides financial, textual, spatial, and temporal data on those awards. The database is the only source of combined information on projects supported by the federal government in the three primary science domains of interest (agricultural harvest, production, and processing of foods; environmental and climate impacts associated with food production and processing; indicators of nutrition and diet-related disease). The database is designed specifically to describe federal science investments and provide empirical data for science policy.

A limitation is that the database does not represent all federal agencies that may be awarding funding for nutrition research (Fleischhacker et al. 2020). Also, not all agencies report funding consistently from year to year. For this reason, reported results are limited to the years between 2016 and 2019; limited data were available for 2020, and the original search identified no relevant project awards from 2020. In addition, the Federal RePORTER is built to provide access to scientific awards to federal agencies and therefore has limitations in its searching capabilities. Fields and advanced query options are limited, and the search string is restricted to 2,500 characters.

\section{Project Methods}

UCS used the Federal RePORTER database to identify federal funding awarded for relevant research projects between 2016 and 2019, as reported by participating federal agencies. Participating federal agencies and years for which complete data have been submitted were obtained from the database are as follows: 
US Department of Defense (DOD)

- Combat Casualty Care Research Program, 2010-2016

- Congressionally Directed Medical Research Programs, 2000-2018

- Traumatic Brain Injury Centre of Excellence, formerly DVBIC, 2000-2016

US Department of Education

- Institution of Education Sciences, 2012-2018

US Department of Health and Human Services (HHS)

- Administration for Children and Families, 2013-2019

- Agency for Healthcare Research and Quality, 2008-2019

- Centers for Disease Control and Prevention, 2008-2019

- Center for Neuroscience and Regenerative Medicine, 2008, 2010-2012, 2014-2017

- Food and Drug Administration, 2008-2019

- National Institute on Disability, Independent Living and Rehabilitation, 2000-2018

- National Institutes of Health, 2008-2019

Environmental Protection Agency (EPA), 2000-2017

National Aeronautics and Space Administration (NASA), 2008-2017

National Science Foundation (NSF), 2000-2018

US Department of Agriculture (USDA)

- Agricultural Research Service, 2000-2019

- Forest Service, 2011-2018

- National Institute of Food and Agriculture, 2008-2016

US Department of Veterans Affairs (VA), 2009-2019

Notably, this list excludes agencies such as the USDA Economic Research Service, which conducts economic research on policy-relevant issues in food, agriculture, and natural resources with an FY 21 budget of \$62 million, as well as potential DOD research efforts to identify environmentally sustainable options for combat rations. The latter was named as a topic of interest in the 2016-2021 National Nutrition Research Roadmap. According to the National Science Foundation Survey of Federal Funds for Research and Development (Table 15: Federal Obligations for Research, by Agency and Performer: FY 2019), 82 agencies across 15 departments had federal obligations for research in FY 2019.

\section{Framework for Project Selection}

The Federal RePORTER database was used to identify relevant scientific project awards from federal agencies between 2016 and 2019. To identify relevant projects in the database, the scope of interest for each of three key domains of sustainable nutrition science was defined as follows:

- Food production includes projects incorporating the harvest, production, or processing of animal products (e.g., beef, pork, poultry), crops (e.g., grains, fruits, vegetables, legumes), and 
wild-caught and farm-raised seafood. All included projects address food intended for human consumption.

- Climate and environment includes projects addressing potential environmental and climate impacts of food production, including waste; loss of biodiversity; heat-trapping emissions; degradation of air, soil, and water; and energy, land, and water use. This domain also includes the potential agricultural impacts of extreme weather (e.g., droughts, floods, extreme heat) that is worsening with climate change.

- Nutrition includes projects addressing human nutrition, including food safety, food security, and diet quality and associated health conditions (e.g., cardiovascular disease, diabetes). This domain does not include health outcomes resulting from environmental exposures, such as farmworker exposure to pesticides or severe weather. The review did not consider drinking water as a food item.

Based on our research framework for sustainable nutrition science (FIGURE A1), which depicts relationships between food production, sustainability, human diets, and human health, the nutrition domain included food safety. However, a safe food supply does not necessarily imply, and can even conflict with, healthy diets and environmentally sustainable food production practices. For example, from a foodborne-illness perspective (health consequences), shelf-stable foods that are highly processed and low in nutritional value might be considered safer than more nutritious perishable foods, while novel approaches or technologies used to improve food safety could result in increased energy use, water use, or food waste (environmental consequences).

Although most funding reported in Federal RePORTER is dedicated to research, funding is also reported for programs, interventions, and educational opportunities (e.g., curricula, conferences, fellowships). We considered these projects within the realm of sustainable nutrition science and included them in the results. Evidence-based programming and educational materials and events contribute to the field of sustainable nutrition science by providing opportunities for fostering knowledge and innovation through learning and cross-disciplinary collaboration.

\section{Search String Development}

We developed the search terms used to identify relevant projects in Federal RePORTER through an iterative process based on the three domains of sustainable nutrition science, informed by two recent reviews of relevant topics: Sparling et al. (2021) and Reinhardt et al. (2020). A set of search strings were then tested in Federal RePORTER through an iterative process: a search string was used to search the database, and potential new terms were identified based on the project terms affiliated with the search results, which are terms (e.g., "healthy diet," "food safety," "urban agriculture") assigned to each project by the project leader and available through Federal RePORTER. The effects of adding each potential new term on the overall search results were then tested one by one. For example, a new term "water quality" would be isolated by adding "water quality" to the search string and removing all other terms from the same domain (climate and environment). The search would then be run with "water quality" and all search terms from the remaining domains (food production; nutrition). If the resulting searches produced new relevant results, the search term was added to the search string. If new results were not relevant to the identified domains based on a review of a sample of 20 project titles and abstracts, the search term was not added. In these cases, potential search terms were identified with similar meaning and greater specificity, with further testing and isolating terms through the process described above. 
Search strings were entered using the "advanced" function for all possible fields (project title, project terms, and project abstract). Subprojects were excluded from the search, as we were interested in identifying total funding levels awarded to projects. Truncation (using the asterisk "*" symbol to truncate the end of a search term, allowing the search engine to return variations on the original word; for example, using the search term "diet*" to return terms such as "diet," "dietary," or "dietitian") functioned only with single-word search terms; truncation was not used in search terms containing multiple words, such as "health disparity." Plurals of all search terms were incorporated through either truncation (single-word search terms) or additional search terms (multiple-word search terms).

\section{Search String and Search Entry Details}

Years: 2016, 2017, 2018, 2019, 2020

Date of search: June 27, 2021

Filters: Exclude subprojects

Search results: 1,068

https://federalreporter.nih.gov/projects/search/?searchId=1eedb234c25045dd91e95240504bf7f6\&search Mode $=$ Advanced\&resultType $=$ projects $\&$ filters $=$

Search string: ("agricultural crop" or "agricultural crops" or "agricultural product" or "agricultural products" or "agricultural production" or "agricultural system" or "agricultural systems" or agroeco* or agroforestry or agronomy or aquaculture or "animal husbandry" or "animal production" or "beef production" or "cattle production" or "crop production" or farm* or fisher* or "food crop" or "food crops" or "food processing" or "food production" or "food system" or "food systems" or horticultur* or livestock or "local food" or "local foods" or meatpacking or "meat packing" or "meat processing" or "pork production" or postharvest or "post-harvest" or "poultry processing" or "poultry production" or "regional food" or "regional foods" or "ruminant production" or "specialty crop" or "specialty crops" or "sustainable agriculture" or "swine production" or "urban agriculture")

AND (diet* or "food insecurity" or "food purchase" or "food purchases" or "food preference" or "food preferences" or "food quality" or "food safety" or "food security" or "food supply" or "good diet" or "good nutrition" or "healthy diet" or "health food" or "health foods" or "healthy eating" or "healthy food" or "healthy foods" or macronutrient* or malnutrition or "Mediterranean diet" or micronutrient* or "nutrient density" or nutrition or nutritional or nutritious or undernutrition or vegan or vegetarian or "Western diet" OR BMI or "body mass index" or "body weight" or cancer or cardiovascular or death or diabetes or disease or endocrine or gastrointestinal or gut or "health status" or healthy or "health disparity" or "health disparities" or "health equity" or "heart disease" or "hypertension" or illness or illnesses or "immune" or "insulin resistance" or metabol* or microbiome or morbidity or mortality or myocardial or obesity or obese or overweight or "quality-adjusted life year" or stroke or unhealthy)

AND ("air quality" or "air pollution" or "algal bloom" or "biodiversity" or "carbon footprint" or climate or "deforestation" or drought or "ecological footprint" or "ecological system" or ecology or ecosystem* or "energy use" or "environmental footprint" or "environmental impact" or flood* "food loss" or "food losses" or "food waste" or "greenhouse effect" or "greenhouse gas" or "greenhouse gases" or "land use" 
or "pollution" or "soil erosion" or "soil health" or "soil quality" or sustainability or sustainable or "water pollution" or "water quality" or "water use")

\section{Inclusion and Exclusion Criteria}

The initial search yielded 1,068 results (FIGURE A2). Three reviewers with expertise relevant to the core domains (nutrition, food production, and climate and environment) reviewed these for inclusion. Two reviewers independently reviewed subsets of 20 projects, one set at a time, until full agreement was reached on a complete set of 20 . After each review period, conflicts were resolved through discussion among all three reviewers and by clarifying the criteria for inclusion and exclusion. This process was repeated three times with a total of 60 project results. One reviewer reviewed the remainder of the projects (1,008 projects); a second reviewer reviewed the final list of included projects (59 projects) to confirm relevance.

Included projects had to meet all of three primary criteria:

- The project explicitly included food production, harvesting, or processing within its scope of work.

- The project included an aspect of nutrition (as described above) within its scope of work.

- The project included an aspect of environmental or climate impacts within its scope of work.

To assess whether these areas were within the scope of work of any project, the abstracts were closely reviewed. Project titles or terms were referenced only to provide greater clarity if the meaning or application of any terms in the abstract was unclear.

Projects were excluded when the abstracts mentioned topics only as introduction or to provide background or context for the research. For example, projects were excluded if they addressed agriculture broadly, without direct implications for food production, or if they addressed nutrition and health only indirectly (e.g., the abstract framed the research as contributing to food security, but the scope of work did not explicitly include assessments of, or direct work on, food security). 


\section{TABLE A1. Search Results \\ Included search results: 59 \\ Unique projects: 53}

The search yielded 59 distinct results, each accompanied by a specified amount of funding granted during a specified fiscal year, and 53 unique projects. These two numbers differ because in some cases, the same research project was granted funding across multiple years or was granted funding multiple times during the same year, each of which would have been considered a separate search result.

\begin{tabular}{|c|c|c|c|c|c|}
\hline Agency & Project Title & $\begin{array}{l}\text { Fiscal } \\
\text { Year }\end{array}$ & Project Number & Recipient & FY Total Cost \\
\hline ARS & $\begin{array}{l}\text { Adding Value to Plant-Based Waste } \\
\text { Materials Through Development of } \\
\text { Novel, Healthy Ingredients and } \\
\text { Functional Foods }\end{array}$ & 2017 & $\begin{array}{l}\text { ARS-0428789- } \\
\text { PROJ }\end{array}$ & $\begin{array}{l}\text { US Agricultural } \\
\text { Research Service }\end{array}$ & $\$ 1,344,016$ \\
\hline ARS & $\begin{array}{l}\text { Adding Value to Plant-Based Waste } \\
\text { Materials Through Development of } \\
\text { Novel, Healthy Ingredients and } \\
\text { Functional Foods }\end{array}$ & 2018 & $\begin{array}{l}\text { ARS-0428789- } \\
\text { PROJ }\end{array}$ & $\begin{array}{l}\text { US Agricultural } \\
\text { Research Service }\end{array}$ & $\$ 1,344,016$ \\
\hline ARS & $\begin{array}{l}\text { Adding Value to Plant-Based Waste } \\
\text { Materials Through Development of } \\
\text { Novel, Healthy Ingredients and } \\
\text { Functional Foods }\end{array}$ & 2019 & $\begin{array}{l}\text { ARS-0428789- } \\
\text { PROJ }\end{array}$ & & $\$ 1,344,016$ \\
\hline ARS & $\begin{array}{l}\text { Detection and Fate of Chemical and } \\
\text { Biological Residues in Food and } \\
\text { Environmental Systems }\end{array}$ & 2019 & $\begin{array}{l}\text { ARS-0430408- } \\
\text { PROJ }\end{array}$ & & $\$ 2,485,692$ \\
\hline NIFA & $\begin{array}{l}120 \text { Committed Beginning Farmers and } \\
\text { Ranchers }\end{array}$ & 2016 & $2016-70017-25345$ & $\begin{array}{l}\text { Arkansas Land and } \\
\text { Community } \\
\text { Development Corp. }\end{array}$ & $\$ 481,080$ \\
\hline NIFA & $\begin{array}{l}\text { Authentic Plant Pollinator Landscape } \\
\text { Research for Educators (Appl-Red) }\end{array}$ & 2016 & $2017-68010-25963$ & $\begin{array}{l}\text { Pennsylvania State } \\
\text { University, } \\
\text { University Park }\end{array}$ & $\$ 144,141$ \\
\hline NIFA & $\begin{array}{l}\text { Breeding Common Bean (Phaseolus } \\
\text { Vulgaris L.) for Resistance to Abiotic } \\
\text { and Biotic Stresses, Sustainable } \\
\text { Production, and Enhanced Nutritional } \\
\text { Value }\end{array}$ & 2016 & 1007384 & $\begin{array}{l}\text { NY Agricultural } \\
\text { Experiment Station }\end{array}$ & \\
\hline NIFA & $\begin{array}{l}\text { CIOA 2- Carrot Improvement for } \\
\text { Organic Agriculture with Added } \\
\text { Grower and Consumer Value }\end{array}$ & 2016 & $2016-51300-25721$ & $\begin{array}{l}\text { US Agricultural } \\
\text { Research Service }\end{array}$ & $\$ 1,999,979$ \\
\hline NIFA & $\begin{array}{l}\text { Cracking Kyphosids: Developing } \\
\text { Culture of High-Value Herbivores for } \\
\text { Sustainable Seafood }\end{array}$ & 2016 & $2016-33610-25456$ & $\begin{array}{l}\text { Kampachi Farms } \\
\text { LLC }\end{array}$ & $\$ 99,740$ \\
\hline NIFA & $\begin{array}{l}\text { Determining Effects of Nitrogen Fixing } \\
\text { Plants on Nutrient Density and } \\
\text { Productivity in Agroforesty and } \\
\text { Polyculture Systems }\end{array}$ & 2016 & 1010123 & $\begin{array}{l}\text { University of the } \\
\text { District of Columbia }\end{array}$ & \\
\hline
\end{tabular}




\begin{tabular}{|c|c|c|c|c|c|}
\hline NIFA & $\begin{array}{l}\text { Developing an Eastern Broccoli } \\
\text { Industry Through Cultivar } \\
\text { Development, Economically and } \\
\text { Environmentally Sustainable } \\
\text { Production and Delivery }\end{array}$ & 2016 & $2016-51181-25402$ & $\begin{array}{l}\text { Cornell University, } \\
\text { Ithaca }\end{array}$ & $\$ 2,019,142$ \\
\hline NIFA & $\begin{array}{l}\text { Economics of the Food Sector: } \\
\text { Consumption, Production, Trade and } \\
\text { Marketing }\end{array}$ & 2016 & 1010309 & $\begin{array}{l}\text { Iowa State } \\
\text { University }\end{array}$ & \\
\hline NIFA & $\begin{array}{l}\text { Educating from the Ground Up: } \\
\text { Promoting Integrated, Experiential } \\
\text { Learning for Resilient Food Systems at } \\
\text { Miami University }\end{array}$ & 2016 & $2017-70001-25988$ & $\begin{array}{l}\text { Miami University, } \\
\text { Oxford }\end{array}$ & $\$ 150,000$ \\
\hline NIFA & $\begin{array}{l}\text { Evaluation of the Prevalence and } \\
\text { Persistence of Shiga Toxin-Producing } \\
\text { E. Coli (STEC) On Organic Mixed } \\
\text { Crop-Livestock Farms That Integrate } \\
\text { Sheep Grazing Within Vegetable Fields }\end{array}$ & 2016 & 1009007 & $\begin{array}{l}\text { University of } \\
\text { California, Davis }\end{array}$ & \\
\hline NIFA & $\begin{array}{l}\text { Exploring Variation in Fish Life } \\
\text { Histories, Population Dynamics and } \\
\text { Community Structure in Aquatic } \\
\text { Ecosystems }\end{array}$ & 2016 & 1007769 & $\begin{array}{l}\text { North Carolina State } \\
\text { University }\end{array}$ & \\
\hline NIFA & $\begin{array}{l}\text { Genomic Tools, Genetic Resources, } \\
\text { and Outreach to Expand Commercial } \\
\text { U.S. Hazelnut Production }\end{array}$ & 2016 & $2016-51181-25412$ & $\begin{array}{l}\text { Oregon State } \\
\text { University }\end{array}$ & $\$ 3,112,410$ \\
\hline NIFA & $\begin{array}{l}\text { Improving Aquaculture`s Value } \\
\text { Through Enhanced Nutrient } \\
\text { Management }\end{array}$ & 2016 & $2016-70007-25758$ & $\begin{array}{l}\text { Auburn University at } \\
\text { Auburn }\end{array}$ & $\$ 326,250$ \\
\hline NIFA & $\begin{array}{l}\text { Improving Sustainable Agriculture } \\
\text { Education at The University of Puerto } \\
\text { Rico Utuado Campus }\end{array}$ & 2016 & $2016-38422-25546$ & $\begin{array}{l}\text { University of Puerto } \\
\text { Rico }\end{array}$ & $\$ 250,000$ \\
\hline NIFA & $\begin{array}{l}\text { Integrated Strategies to Mitigate } \\
\text { Environmental Stresses in Vegetable } \\
\text { Crops }\end{array}$ & 2016 & 1008386 & $\begin{array}{l}\text { Texas A\&M } \\
\text { University }\end{array}$ & \\
\hline NIFA & $\begin{array}{l}\text { Island Fresh Student Enterprises, } \\
\text { Southeast Island School District }\end{array}$ & 2016 & $2016-38414-25876$ & $\begin{array}{l}\text { Southeast Island } \\
\text { School District }\end{array}$ & $\$ 45,471$ \\
\hline NIFA & $\begin{array}{l}\text { Multifunctional Agroforestry as a } \\
\text { Sustainable Food Production Option in } \\
\text { Illinois }\end{array}$ & 2016 & 1007823 & University of Illinois & \\
\hline NIFA & $\begin{array}{l}\text { Multi-Regional Risk Analysis of Farm } \\
\text { Manure Use: Balancing Soil Health and } \\
\text { Food Safety for Organic Fresh Produce } \\
\text { Production }\end{array}$ & 2016 & $2016-51300-25724$ & $\begin{array}{l}\text { University of } \\
\text { California, Davis }\end{array}$ & $\$ 1,999,848$ \\
\hline NIFA & $\begin{array}{l}\text { Organic Crop and Forage Rotations for } \\
\text { Dairy Production Systems }\end{array}$ & 2016 & 1006998 & $\begin{array}{l}\text { University of } \\
\text { Minnesota }\end{array}$ & \\
\hline NIFA & $\begin{array}{l}\text { Preserving Choctaw Culture by } \\
\text { Growing Hope }\end{array}$ & 2016 & $2016-33800-25594$ & $\begin{array}{l}\text { Choctaw Nation of } \\
\text { Oklahoma }\end{array}$ & $\$ 399,864$ \\
\hline NIFA & $\begin{array}{l}\text { Rebuilding Local Food Systems in } \\
\text { Farmworker Communities Project }\end{array}$ & 2016 & $2016-33800-25589$ & $\begin{array}{l}\text { Farmworker } \\
\text { Association of } \\
\text { Florida, Inc. }\end{array}$ & $\$ 500,000$ \\
\hline
\end{tabular}




\begin{tabular}{|c|c|c|c|c|c|}
\hline NIFA & $\begin{array}{l}\text { Smart Cities and Connected } \\
\text { Communities: A Workshop Bridging } \\
\text { Rural, Peri-Urban and Urban } \\
\text { Sustainability }\end{array}$ & 2016 & $2016-38831-25863$ & $\begin{array}{l}\text { University of the } \\
\text { District of Columbia }\end{array}$ & $\$ 25,000$ \\
\hline NIFA & $\begin{array}{l}\text { Sources of Methionine for Organically- } \\
\text { Fed Poultry }\end{array}$ & 2016 & 1008091 & $\begin{array}{l}\text { University of } \\
\text { California, Davis }\end{array}$ & \\
\hline NIFA & $\begin{array}{l}\text { Strengthening US Agriculture with } \\
\text { Multidisciplinary International } \\
\text { Undergraduate Research and Extension } \\
\text { Experiences }\end{array}$ & 2016 & $2017-67032-26014$ & $\begin{array}{l}\text { University of } \\
\text { Tennessee }\end{array}$ & $\$ 281,236$ \\
\hline NIFA & $\begin{array}{l}\text { Understanding the Ecology of Shellfish } \\
\text { and Their Pathogens to Improve } \\
\text { Shellfish Management and Production }\end{array}$ & 2016 & 1009201 & $\begin{array}{l}\text { Rutgers, The State } \\
\text { University of NJ } \\
\text { New Brunswick }\end{array}$ & \\
\hline NIFA & $\begin{array}{l}\text { Winnebago Community Food System } \\
\text { Planning Project }\end{array}$ & 2016 & $2016-33800-25583$ & $\begin{array}{l}\text { Ho-Chunk } \\
\text { Community } \\
\text { Development } \\
\text { Corporation }\end{array}$ & $\$ 34,019$ \\
\hline NIH & $\begin{array}{l}\text { 18th International Conference on } \\
\text { Harmful Algae }\end{array}$ & 2018 & 1R13ES029388-01 & $\begin{array}{l}\text { Woods Hole } \\
\text { Oceanographic } \\
\text { Institution }\end{array}$ & $\$ 8,000$ \\
\hline NIH & $\begin{array}{l}\text { Greater Caribbean Center for Ciguatera } \\
\text { Research }\end{array}$ & 2018 & 1P01ES028949-01 & $\begin{array}{l}\text { Florida Gulf Coast } \\
\text { University }\end{array}$ & $\$ 493,091$ \\
\hline NIH & $\begin{array}{l}\text { Greater Caribbean Center for Ciguatera } \\
\text { Research }\end{array}$ & 2019 & 5P01ES028949-02 & $\begin{array}{l}\text { Florida Gulf Coast } \\
\text { University }\end{array}$ & $\$ 471,042$ \\
\hline NIH & $\begin{array}{l}\text { Impact of El Nino on Environmental } \\
\text { Mercury and Human Exposure }\end{array}$ & 2016 & 1R21ES026960-01 & Duke University & $\$ 159,500$ \\
\hline NIH & $\begin{array}{l}\text { Impact of El Nino on Environmental } \\
\text { Mercury and Human Exposure }\end{array}$ & 2017 & 5R21ES026960-02 & Duke University & $\$ 183,833$ \\
\hline NIH & $\begin{array}{l}\text { Natural Sources and Microbial } \\
\text { Transformation of Marine Halogenated } \\
\text { Pollutants }\end{array}$ & 2018 & 1R01ES030316-01 & $\begin{array}{l}\text { University of } \\
\text { California, San } \\
\text { Diego }\end{array}$ & $\$ 125,550$ \\
\hline NIH & $\begin{array}{l}\text { Natural Sources and Microbial } \\
\text { Transformation of Marine Halogenated } \\
\text { Pollutants }\end{array}$ & 2019 & 5R01ES030316-02 & $\begin{array}{l}\text { University of } \\
\text { California, San } \\
\text { Diego }\end{array}$ & $\$ 125,550$ \\
\hline NSF & $\begin{array}{l}\text { Belmont Forum Collaborative Research } \\
\text { Food-Water-Energy Nexus: The } \\
\text { Moveable Nexus: Design-Led Urban } \\
\text { Food, Water and Energy Management } \\
\text { Innovation in New Boundary } \\
\text { Conditions of Change }\end{array}$ & 2018 & 1832214 & $\begin{array}{l}\text { University of } \\
\text { Michigan at Ann } \\
\text { Arbor }\end{array}$ & $\$ 76,139$ \\
\hline NSF & $\begin{array}{l}\text { Career: Mapping Pathways to Food } \\
\text { Security and Sustainable Development }\end{array}$ & 2018 & 1750621 & $\begin{array}{l}\text { University of } \\
\text { California, Davis }\end{array}$ & $\$ 433,988$ \\
\hline NSF & $\begin{array}{l}\text { Careers in Food, Energy, and the } \\
\text { Environment-An Interdisciplinary } \\
\text { Approach }\end{array}$ & 2016 & 1643833 & $\begin{array}{l}\text { Michigan State } \\
\text { University }\end{array}$ & $\$ 999,983$ \\
\hline NSF & $\begin{array}{l}\text { CNH-L: Interactive Dynamics of Reef } \\
\text { Fisheries and Human Health }\end{array}$ & 2018 & 1826668 & Harvard University & $\$ 1,359,998$ \\
\hline
\end{tabular}




\begin{tabular}{|c|c|c|c|c|c|}
\hline NSF & $\begin{array}{l}\text { CNH-L: The Influence of Conflicting } \\
\text { Policies and Supply-Chain Pressures on } \\
\text { Farmers' Decisions and Tradeoffs with } \\
\text { Respect to Biodiversity, Profitability, } \\
\text { and Sustainability }\end{array}$ & 2018 & 1824871 & $\begin{array}{l}\text { University of } \\
\text { California, Berkeley }\end{array}$ & $\$ 1,301,737$ \\
\hline NSF & $\begin{array}{l}\text { CNH-S: Socio-Economic Factors, Land } \\
\text { and Water Quality, and the Dynamics } \\
\text { Between Rural and Urban Zones of } \\
\text { Food Production and Consumption. }\end{array}$ & 2018 & 1824949 & $\begin{array}{l}\text { North Carolina } \\
\text { Agricultural and } \\
\text { Technical State } \\
\text { University }\end{array}$ & $\$ 749,989$ \\
\hline NSF & $\begin{array}{l}\text { EAGER: FEWSTERN: US-China } \\
\text { Food-Energy-Water Systems } \\
\text { Transdisciplinary Environmental } \\
\text { Research Network }\end{array}$ & 2017 & 1739474 & $\begin{array}{l}\text { University of } \\
\text { Tennessee }\end{array}$ & $\$ 150,000$ \\
\hline NSF & $\begin{array}{l}\text { Greater Caribbean Center for Ciguatera } \\
\text { Research }\end{array}$ & 2018 & 1841811 & $\begin{array}{l}\text { Florida Gulf Coast } \\
\text { University }\end{array}$ & $\$ 739,638$ \\
\hline NSF & $\begin{array}{l}\text { Hazards SEES: Understanding Cross- } \\
\text { scale Interactions of Trade and Food } \\
\text { Policy to Improve Resilience to } \\
\text { Drought Risk }\end{array}$ & 2018 & 1832393 & Clark University & $\$ 1,563,153$ \\
\hline NSF & $\begin{array}{l}\text { INFEWS/T1: Intensification in the } \\
\text { World's Largest Agricultural Frontier: } \\
\text { Integrating Food Production, Water } \\
\text { Use, Energy Demand, and } \\
\text { Environmental Integrity in a Changing } \\
\text { Climate }\end{array}$ & 2017 & 1739724 & $\begin{array}{l}\text { Woods Hole } \\
\text { Research Center, } \\
\text { Inc. }\end{array}$ & $\$ 828,428$ \\
\hline NSF & $\begin{array}{l}\text { INFEWS/T1: Linking Current and } \\
\text { Future Hydrologic Change to } \\
\text { Hydropower, Human Nutrition, and } \\
\text { Livelihoods in the Lower Mekong } \\
\text { Basin }\end{array}$ & 2017 & 1740042 & $\begin{array}{l}\text { Arizona State } \\
\text { University, Tempe } \\
\text { Campus }\end{array}$ & $\$ 1,331,133$ \\
\hline NSF & $\begin{array}{l}\text { International Workshop on the Nexus } \\
\text { of Food, Energy, Water and Soil }\end{array}$ & 2016 & 1649510 & $\begin{array}{l}\text { University of } \\
\text { Tennessee }\end{array}$ & $\$ 20,000$ \\
\hline NSF & $\begin{array}{l}\text { NRT-INFEWS: Integrated Urban } \\
\text { Solutions for Food, Energy, and Water } \\
\text { Management }\end{array}$ & 2017 & 1735325 & $\begin{array}{l}\text { University of } \\
\text { California, Los } \\
\text { Angeles }\end{array}$ & $\$ 3,000,000$ \\
\hline NSF & $\begin{array}{l}\text { Planning IUCRC at Georgia Institute of } \\
\text { Technology: Center for Engineering } \\
\text { and Manufacturing Technologies } \\
\text { Advancing Food Safety and Security } \\
\text { (CAFSS) }\end{array}$ & 2018 & 1822117 & $\begin{array}{l}\text { Georgia Tech } \\
\text { Applied Research } \\
\text { Corporation }\end{array}$ & $\$ 15,000$ \\
\hline NSF & $\begin{array}{l}\text { PPSR: Workshop on Citizen Science in } \\
\text { Agrifood Systems: Exploring the } \\
\text { Synergy Between Science and Counter- } \\
\text { Expertise }\end{array}$ & 2017 & 1743138 & $\begin{array}{l}\text { University of Hawaii } \\
\text { at Manoa }\end{array}$ & $\$ 32,929$ \\
\hline NSF & $\begin{array}{l}\text { RII Track-2 FEC: Sustainable Socio- } \\
\text { Economic, Ecological, and } \\
\text { Technological Scenarios for Achieving } \\
\text { Global Climate Stabilization Through } \\
\text { Negative CO2 Emission Policies }\end{array}$ & 2016 & 1632810 & $\begin{array}{l}\text { Montana State } \\
\text { University, Bozeman }\end{array}$ & $\$ 6,000,000$ \\
\hline NSF & $\begin{array}{l}\text { SBIR Phase I: Modular Ozonation } \\
\text { Technology for Improved Quality and } \\
\text { Reduced Spoilage from Farm to Retail }\end{array}$ & 2016 & 1622071 & Surfplasma, Inc. & $\$ 224,999$ \\
\hline
\end{tabular}




\begin{tabular}{|c|c|c|c|c|c|}
\hline NSF & $\begin{array}{l}\text { SCC-RCN: Developing an } \\
\text { Informational Infrastructure for } \\
\text { Building Smart Regional Foodsheds }\end{array}$ & 2017 & 1737573 & $\begin{array}{l}\text { University of } \\
\text { California, Davis }\end{array}$ & $\$ 500,000$ \\
\hline NSF & $\begin{array}{l}\text { Smart Cities and Connected } \\
\text { Communities: A Workshop Bridging } \\
\text { Rural, Peri-Urban and Urban } \\
\text { Sustainability }\end{array}$ & 2017 & 1712678 & $\begin{array}{l}\text { University of the } \\
\text { District of Columbia }\end{array}$ & $\$ 25,000$ \\
\hline NSF & $\begin{array}{l}\text { The Effects of Climate and Population } \\
\text { Density on Agricultural Production }\end{array}$ & 2018 & 1749059 & University of Utah & $\$ 215,894$ \\
\hline NSF & $\begin{array}{l}\text { WSC-Category } 2 \text { Collaborative: } \\
\text { Impacts of Agricultural Decision } \\
\text { Making and Adaptive Management on } \\
\text { Food Security }\end{array}$ & 2018 & 1801251 & $\begin{array}{l}\text { University of } \\
\text { California, Santa } \\
\text { Barbara }\end{array}$ & $\$ 628,777$ \\
\hline NSF & $\begin{array}{l}\text { WSC-Category } 2 \text { Collaborative: } \\
\text { Impacts of Agricultural Decision } \\
\text { Making and Adaptive Management on } \\
\text { Food Security }\end{array}$ & 2018 & 1830752 & $\begin{array}{l}\text { University of } \\
\text { Arizona }\end{array}$ & $\$ 980,887$ \\
\hline
\end{tabular}


TABLE A2. Funding Estimates, by Year (Figure 2 data)

Federal Investments in All Research, Nutrition Research, and Sustainable Nutrition Science, FY 2016 -2019 (millions USD)

\begin{tabular}{|c|c|c|c|c|c|c|}
\hline & 2016 & 2017 & 2018 & 2019 & Total & Average \\
\hline All federal research & $\$ 67,105$ & $\$ 69,871$ & $\$ 74,588$ & $\$ 81,188$ & $\$ 292,752$ & $\$ 73,188$ \\
\hline $\begin{array}{l}\text { Research obligations for } \\
\text { ICHNR } \\
\text { agency/department }\end{array}$ & $\$ 48,081$ & $\$ 50,056$ & $\$ 52,903$ & $\$ 58,226$ & $\$ 209,265$ & $\$ 52,316$ \\
\hline $\begin{array}{l}\text { All funding reported in } \\
\text { Federal RePORTER }\end{array}$ & $\$ 38,846$ & $\$ 38,232$ & $\$ 41,044$ & $\$ 37,295$ & $\$ 155,417$ & $\$ 38,854$ \\
\hline Nutrition research & -- & -- & -- & $\$ 1,900$ & -- & $\$ 1,900$ \\
\hline $\begin{array}{l}\text { Sustainable nutrition } \\
\text { science (reported) }\end{array}$ & $\$ 19.3$ & $\$ 7.4$ & $\$ 10$ & $\$ 4.4$ & $\$ 41.1$ & $\$ 10.3$ \\
\hline $\begin{array}{l}\text { Sustainable nutrition } \\
\text { science (projected) }\end{array}$ & $\mathrm{N} / \mathrm{A}$ & $\$ 20.1$ & $\$ 21.5$ & $\$ 23.3$ & $\$ 84.2$ & $\$ 21.1$ \\
\hline $\begin{array}{l}\text { Sustainable nutrition } \\
\text { science (estimated) }\end{array}$ & N/A & $\$ 13.8$ & $\$ 15.8$ & $\$ 13.9$ & $\$ 62.8$ & $\$ 15.7$ \\
\hline
\end{tabular}

Total federal research funding and research funding at Interagency Committee on Human Nutrition Research (ICHNR) agencies and departments are based on basic and applied research obligations for fiscal years 2016-2019. Federal nutrition research budget based on Fleischhacker et al. (2020) estimates. Sustainable nutrition science funding (reported) is based on Federal RePORTER data and may be an underestimate due to inconsistent reporting to Federal RePORTER by agencies. Sustainable nutrition science funding (projected) is based on 2016 sustainable nutrition science funding (reported) with an annual increase consistent with increases across all federal research funding. These values are likely overestimates: Fleischhacker et al. (2020) demonstrated that nutrition research funding has not increased at the pace of all federal research. Sustainable nutrition science funding (estimated) is the midpoint of reported and projected values.

SOURCES: NCES (2021); NIH (2021); FLEISCHHACKER ET AL. (2020). 


\section{TABLE A3. Federal Recommendations for Sustainable Nutrition Science, 2010-}

\section{1}

Since 2010, more than a dozen federal reports have included recommendations to advance research and education at the intersection of nutrition, food production, and climate and environment.

\begin{tabular}{|c|c|c|c|}
\hline Year & Institution & Publication & Summary of Relevant Recommendations \\
\hline 2010 & $\begin{array}{l}\text { National } \\
\text { Research } \\
\text { Council }\end{array}$ & $\begin{array}{l}\text { Toward Sustainable } \\
\text { Agricultural Systems } \\
\text { in the 21st Century: } \\
\text { Consensus Study } \\
\text { Report }\end{array}$ & $\begin{array}{l}\text { To pursue systemic changes in farming systems, research and } \\
\text { development has to address multiple dimensions of sustainability, } \\
\text { including productivity and environmental, economic, and social } \\
\text { sustainability. }\end{array}$ \\
\hline 2012 & $\begin{array}{l}\text { President's } \\
\text { Council of } \\
\text { Advisors on } \\
\text { Science and } \\
\text { Technology }\end{array}$ & $\begin{array}{l}\text { 2012 Report to the } \\
\text { President on } \\
\text { Agricultural } \\
\text { Preparedness and the } \\
\text { Agriculture Research } \\
\text { Enterprise }\end{array}$ & $\begin{array}{l}\text { Public investors in agricultural research at the federal, state, and local } \\
\text { levels should organize research and development agendas around } \\
\text { seven key challenges: managing new pests, pathogens, and invasive } \\
\text { plants; increasing the efficiency of water use; reducing the } \\
\text { environmental footprint of agriculture; growing food in a changing } \\
\text { climate; managing the production of bioenergy; producing safe and } \\
\text { nutritious food; and assisting with global food security and } \\
\text { maintaining abundant yields. }\end{array}$ \\
\hline 2014 & $\begin{array}{l}\text { Institute of } \\
\text { Medicine }\end{array}$ & $\begin{array}{l}\frac{\text { Sustainable Diets: }}{\text { Food for Healthy }} \\
\text { People and a Healthy } \\
\text { Planet: Workshop } \\
\text { Summary }\end{array}$ & $\begin{array}{l}\text { Conduct more interdisciplinary and transdisciplinary systems } \\
\text { research encompassing multiple dimensions of sustainability and } \\
\text { resiliency, including the social costs of food systems; linkages } \\
\text { between soil quality and human health; role of animal agriculture in } \\
\text { sustainable diets and ecosystems; food system scale (e.g., local, } \\
\text { regional) and market concentration; effects of geography, } \\
\text { seasonality, storage, and processing on food quality and } \\
\text { environmental outcomes; and consumer behavior change strategies. }\end{array}$ \\
\hline 2015 & $\begin{array}{l}\text { Institute of } \\
\text { Medicine }\end{array}$ & $\begin{array}{l}\text { A Framework for } \\
\text { Assessment Effects of } \\
\text { the Food System }\end{array}$ & $\begin{array}{l}\text { Congress and federal agencies should fund and support food systems } \\
\text { data collection and continue efforts to share data and develop public- } \\
\text { private mechanisms for collaboration. Scientists in academia, the } \\
\text { private sector, and government agencies should be trained in all } \\
\text { aspects of complex systems approaches - including systems research } \\
\text { design, data collection, and analytical methodologies - and the use of } \\
\text { models. }\end{array}$ \\
\hline 2015 & $\begin{array}{l}2015 \text { Dietary } \\
\text { Guidelines } \\
\text { Advisory } \\
\text { Committee }\end{array}$ & $\begin{array}{l}\text { Scientific Report of the } \\
\text { 2015 Dietary } \\
\text { Guidelines Advisory } \\
\text { Committee }\end{array}$ & $\begin{array}{l}\text { Develop and test communication strategies to help motivate people } \\
\text { of all ages to consume increasingly sustainable diets; develop and } \\
\text { test systems to ensure that sustainable diets are affordable and } \\
\text { available to all populations; develop more in-depth analysis of US } \\
\text { dietary patterns and enhanced environmental sustainability with } \\
\text { different animal production regimens; develop updated analysis of } \\
\text { environmental sustainability of dietary patterns with respect to fish } \\
\text { consumption, including nutrient profiles and production regimens. }\end{array}$ \\
\hline 2016 & $\begin{array}{l}\text { Interagency } \\
\text { Committee } \\
\text { on Human } \\
\text { Nutrition } \\
\text { Research }\end{array}$ & $\begin{array}{l}\frac{\text { National Nutrition }}{\text { Research Roadmap, }} \\
\underline{2016-2021}\end{array}$ & $\begin{array}{l}\text { Identify effective approaches to enhance the environmental } \\
\text { sustainability of healthy eating patterns through interdisciplinary } \\
\text { research, focusing on such topics as: biodiversity and energy, land } \\
\text { and water use associated with healthy diets; promotion and } \\
\text { maintenance of dietary diversity; consumer acceptance and economic } \\
\text { viability of environmental policies and practices; consumer, } \\
\text { institutional, and retailer practices that could reduce food waste; } \\
\text { impacts of local food production and comparisons across production } \\
\text { systems; and impacts on agricultural production and food distribution } \\
\text { of a population-wide transition to a healthy diet. }\end{array}$ \\
\hline 2017 & $\begin{array}{l}\text { National } \\
\text { Academies of } \\
\text { Science, }\end{array}$ & $\begin{array}{l}\text { Redesigning the } \\
\text { Process for } \\
\text { Establishing the } \\
\text { Dietary Guidelines }\end{array}$ & $\begin{array}{l}\text { Adding complex systems approaches to current analytical approaches } \\
\text { used in the Dietary Guidelines for Americans process can advance } \\
\text { the understanding of complex interrelated factors at play in both } \\
\text { population and individual health. Establishing and modeling the }\end{array}$ \\
\hline
\end{tabular}




\begin{tabular}{|c|c|c|c|}
\hline & $\begin{array}{l}\text { Engineering, } \\
\text { and Medicine }\end{array}$ & & $\begin{array}{l}\text { multisectoral relationships and pathways between diet and health can } \\
\text { strengthen the science base of dietary guidance and identify } \\
\text { important gaps that require further investigation. }\end{array}$ \\
\hline 2019 & $\begin{array}{l}\text { National } \\
\text { Academies of } \\
\text { Sciences, } \\
\text { Engineering, } \\
\text { and Medicine }\end{array}$ & $\begin{array}{l}\text { Sustainable Diets, } \\
\text { Food, and Nutrition: } \\
\text { Proceedings of a } \\
\text { Workshop }\end{array}$ & $\begin{array}{l}\text { Address gaps in understanding what constitutes a sustainable diet for } \\
\text { different populations and how such diets are best measured in } \\
\text { different contexts; develop guidance on the economic impact of } \\
\text { sustainable diets for all actors in the food chain; and provide policy } \\
\text { analysis to better integrate sustainability issues into nutrition policy. }\end{array}$ \\
\hline 2019 & $\begin{array}{l}\text { National } \\
\text { Academies of } \\
\text { Sciences, } \\
\text { Engineering, } \\
\text { and Medicine }\end{array}$ & $\begin{array}{l}\text { Breakthroughs to } \\
\text { Advance Food and } \\
\text { Agricultural Research } \\
\text { by } 2030\end{array}$ & $\begin{array}{l}\text { Major goals for food and agricultural research by } 2030 \text { include } \\
\text { improving the efficiency of food and agricultural systems; increasing } \\
\text { the sustainability of agriculture; and increasing the resiliency of } \\
\text { agricultural systems to adapt to rapid changes and extreme } \\
\text { conditions. Research challenges include increasing nutrient use } \\
\text { efficiency and mobilizing genetic diversity in crops; reducing soil } \\
\text { loss and degradation; optimizing water use; improving animal } \\
\text { genetics; developing precision livestock systems; early detection of } \\
\text { plant and animal disease, as well as foodborne pathogens; and } \\
\text { reducing waste and loss throughout the supply chain. }\end{array}$ \\
\hline 2020 & $\begin{array}{l}2020 \text { Dietary } \\
\text { Guidelines } \\
\text { Advisory } \\
\text { Committee }\end{array}$ & $\begin{array}{l}\text { Scientific Report of the } \\
\begin{array}{l}\text { 2020 Dietary } \\
\text { Guidelines Advisory } \\
\text { Committee }\end{array}\end{array}$ & $\begin{array}{l}\text { Research is needed to develop a systems approach that is relevant to } \\
\text { the Dietary Guidelines for Americans. Long-term maintenance of } \\
\text { healthy food and beverage intakes requires long-term support of } \\
\text { associated food systems. The committee's review reinforces the need } \\
\text { to consider the Dietary Guidelines for Americans in the context of } \\
\text { the food environment and the overall food system, including the } \\
\text { sustainability of the food supply. }\end{array}$ \\
\hline 2020 & $\begin{array}{l}\text { National } \\
\text { Academies of } \\
\text { Sciences, } \\
\text { Engineering, } \\
\text { and Medicine }\end{array}$ & 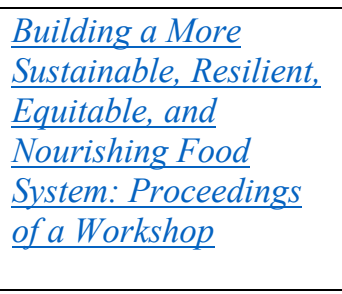 & $\begin{array}{l}\text { Research priorities include agroecology; circular food systems; } \\
\text { improving nutrition, food safety, and environmental impacts of } \\
\text { aquaculture; and socioeconomic research on how to implement } \\
\text { needed food systems changes, including public policy changes and } \\
\text { farmer adoption of more sustainable farming practices. } \\
\text { Interdisciplinary funding is important for implementing the priorities } \\
\text { identified. }\end{array}$ \\
\hline 2021 & $\begin{array}{l}\text { National } \\
\text { Academies of } \\
\text { Sciences, } \\
\text { Engineering, } \\
\text { and Medicine }\end{array}$ & $\begin{array}{l}\text { Creating the Future } \\
\text { Workforce in Food, } \\
\text { Agriculture, and } \\
\text { Natural Resources: } \\
\text { Proceedings of a } \\
\text { Workshop }\end{array}$ & $\begin{array}{l}\text { Workforce development in food, agriculture, and natural resources } \\
\text { can be supported by: providing greater public funding for agricultural } \\
\text { research to remain competitive with international industries and } \\
\text { institutions; establishing training grants to encourage } \\
\text { interdisciplinary research; partnering with industry to provide } \\
\text { transformative research experiences for students; and expanding } \\
\text { research opportunities and subjects across all education levels. }\end{array}$ \\
\hline 2021 & $\begin{array}{l}\text { National } \\
\text { Academies of } \\
\text { Sciences, } \\
\text { Engineering, } \\
\text { and Medicine }\end{array}$ & $\begin{array}{l}\text { The Challenge of } \\
\text { Feeding the World } \\
\text { Sustainably: Summary } \\
\text { of the US-UK } \\
\text { Scientific Forum on } \\
\text { Sustainable } \\
\text { Agriculture }\end{array}$ & $\begin{array}{l}\text { Research gaps include: supply chains, the economics of distribution } \\
\text { systems, and the need to minimize waste; synergies among carbon } \\
\text { sequestration, biodiversity conservation, and sustainable agriculture, } \\
\text { as well as synergies and conflicts in land use; policy levers for } \\
\text { change, based on multidisciplinary, coordinated, and international } \\
\text { research; and applications of biotechnology, implementation of } \\
\text { integrated best management, and improved understanding of the soil } \\
\text { microbiome to enhance crop performance and soil carbon storage. }\end{array}$ \\
\hline
\end{tabular}




\section{FIGURE A1. Framework for Sustainable Nutrition Science}

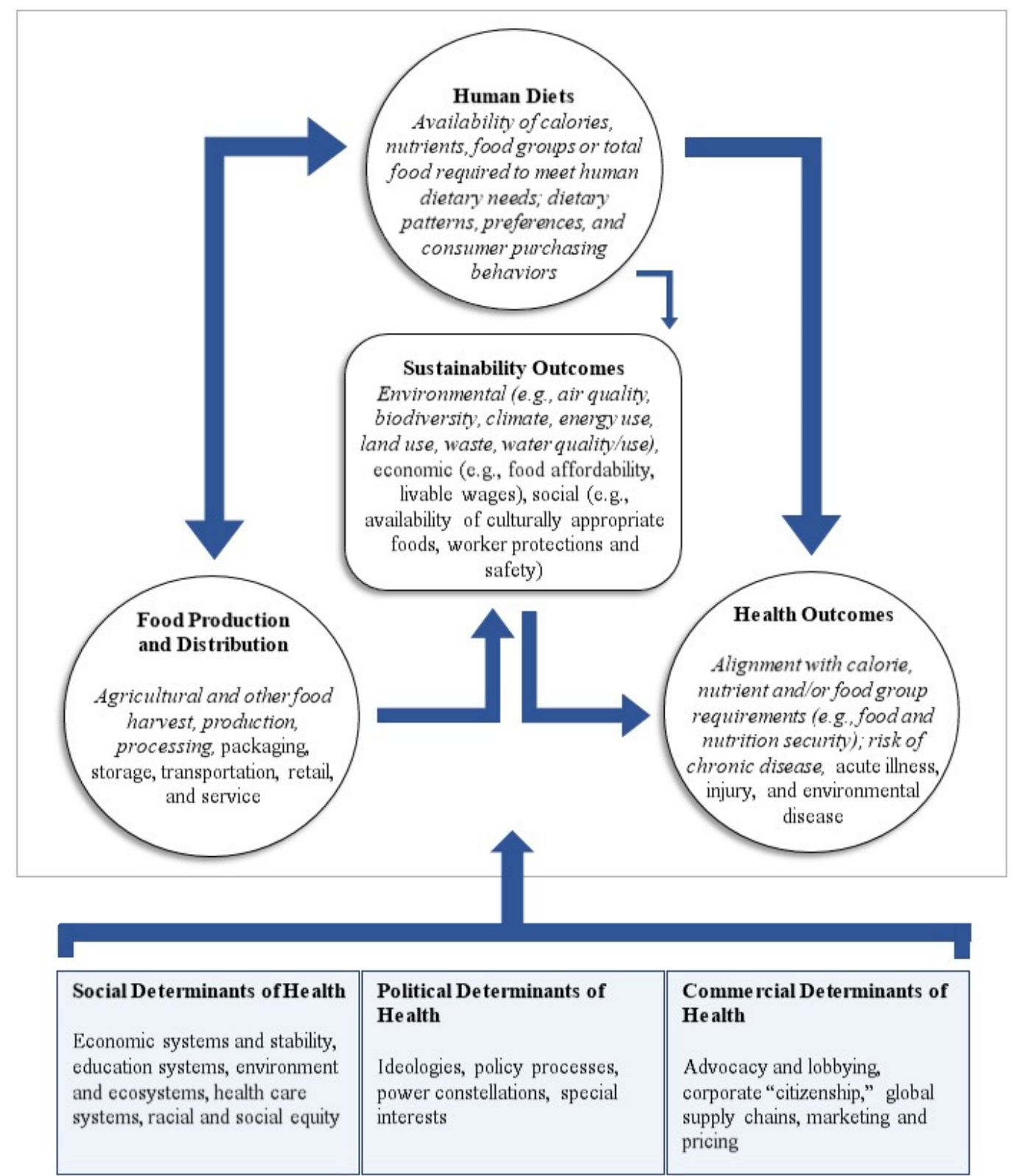

SOURCE: Adapted from Reinhardt et al. (2020) and Tagtow, Herman, andSabo (2021)

From Silos to Systems defines sustainable nutrition science as research and education at the intersection of nutrition, food production, and climate and environment. Sustainable nutrition science could be considered a subset of the broader field of sustainable food systems research, which would also incorporate points along the supply chain beyond food production, including food packaging, storage, transportation, retail, and service; economic and social dimensions of sustainability; and illness or injury resulting from environmental exposures or workplace hazards related to food production. Sustainable food systems research may also incorporate the social, political, and commercial determinants of food systems and health. FIGURE A1 depicts the field of sustainable nutrition science, indicated in italics, in the context of sustainable food systems research.

SOURCES: TAGTOW, HERMAN, AND CUNNINGHAM-SABO (2021); SPIKER, REINHARDT, AND BRUENING (2020); HLPE (2014). 


\section{FIGURE A2. PRISMA Flow Diagram for Project Search and Selection}

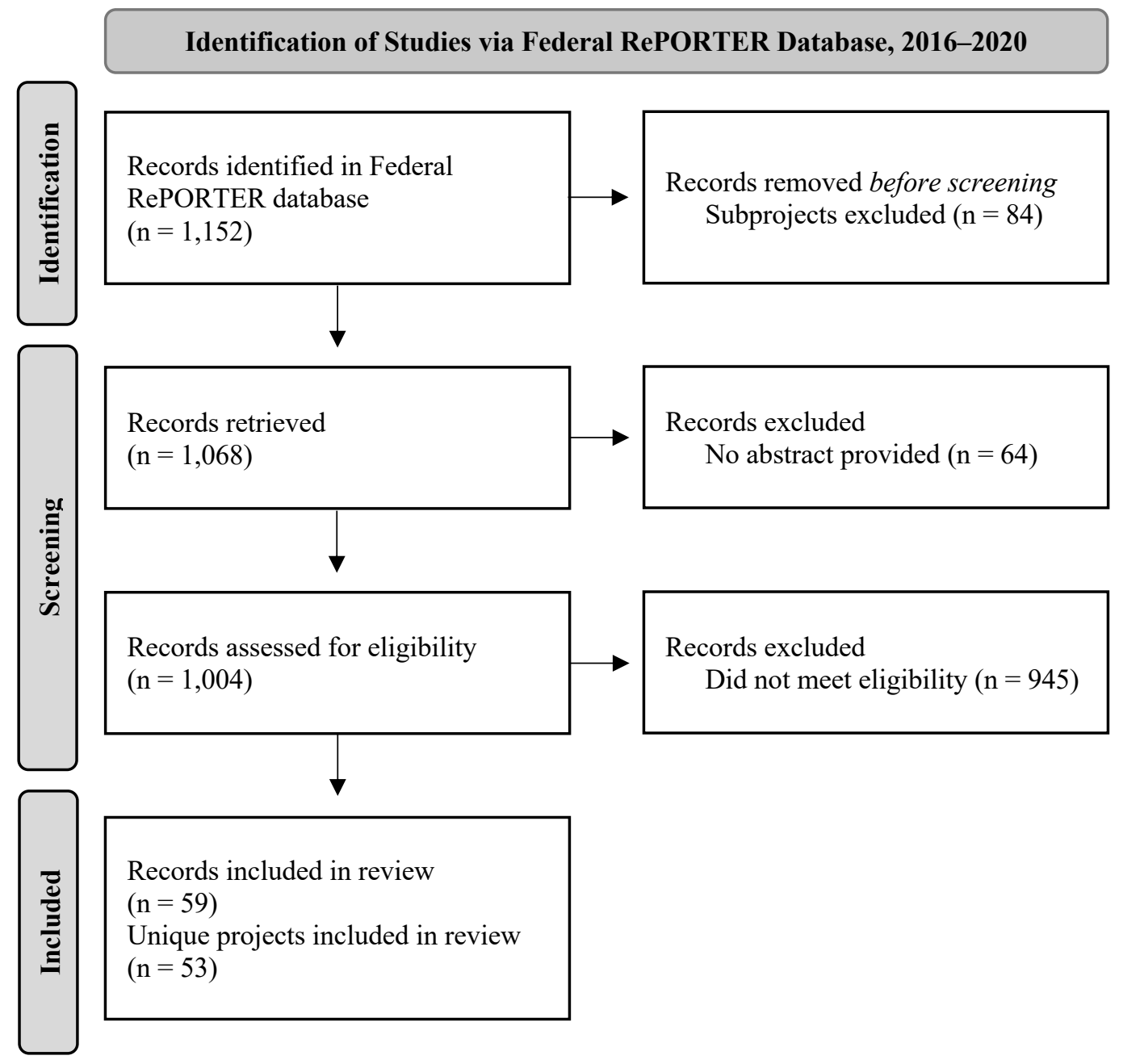

Unique projects excluded duplicate Federal RePORTER records resulting from funding distributed across multiple fiscal years for the same project. In such cases, all funding distributed was still counted in total funding.

SOURCE: PAGE ET AL. (2021). 


\section{References}

Fleischhacker, Sheila E., Catherine E. Woteki, Paul M. Coates, Van S. Hubbard, Grace E. Flaherty, Daniel R. Glickman, Thomas R. Harkin, et al. 2020. "Strengthening National Nutrition Research: Rationale and Options for a New Coordinated Federal Research Effort and Authority." American Journal of Clinical Nutrition 112 (3): 721-69. https://doi.org/10.1093/ajen/nqaa179

HLPE (High Level Panel of Experts on Food Security and Nutrition). 2014. "Food Losses and Waste in the Context of Sustainable Food Systems.” Rome: Food and Agriculture Organization. http://www.fao.org/3/i3901e/i3901e.pdf

ICHNR (Interagency Committee on Human Nutrition Research). 2016. National Nutrition Research Roadmap 2016-2021: Advancing Nutrition Research to Improve and Sustain Health. Washington, DC: Interagency Committee on Human Nutrition Research.

NCSES (National Center for Science and Engineering Statistics). 2021. "Data Table: Federal Obligations for Research, by Agency and Performer." FFRDC Research and Development Survey. Alexandria, VA: National Science Foundation. https://www.nsf.gov/statistics/srvyffrdc

NIH (National Institutes of Health). 2021. "Federal RePORTER.” Bethesda, MD: US Department of Health and Human Services. https://federalreporter.nih.gov

Page, Matthew J., Joanne E. McKenzie, Patrick M. Bossuyt, Isabelle Boutron, Tammy C. Hoffmann, Cynthia D. Mulrow, Larissa Shamseer, et al. 2021. "The PRISMA 2020 Statement: An Updated Guideline for Reporting Systematic Reviews.” BMJ 372 (71). https://doi.org/10.1136/bmj.n71

Reinhardt, Sarah L., Rebecca Boehm, Nicole Tichenor Blackstone, Naglaa H. El-Abbadi, Joy S. McNally Brandow, Salima F. Taylor, and Marcia S. DeLonge. 2020. "Systematic Review of Dietary Patterns and Sustainability in the United States." Advances in Nutrition 11 (4): 1016-31. https://doi.org/10.1093/advances/nmaa026

Sparling, Thalia M, Howard White, Samuel Boakye, Denny John, and Suneetha Kadiyala. 2021. "Understanding Pathways Between Agriculture, Food Systems, and Nutrition: An Evidence and Gap Map of Research Tools, Metrics, and Methods in the Last 10 Years." Advances in Nutrition 12 (4): 1122-1136. https://doi.org/10.1093/advances/nmaa158

Spiker, Marie, Sarah Reinhardt, and Meg Bruening. 2020. "Academy of Nutrition and Dietetics: Revised 2020 Standards of Professional Performance for Registered Dietitian Nutritionists (Competent, Proficient, and Expert) in Sustainable, Resilient, and Healthy Food and Water Systems." Journal of the Academy of Nutrition and Dietetics 120 (9): 1568-1585.e28. https://doi.org/10.1016/j.jand.2020.05.010

Tagtow, Angela, Dena Herman, and Leslie Cunningham-Sabo. 2021. "Next-Generation Solutions to Address Adaptive Challenges in Dietetics Practice: The I+PSE Conceptual Framework for Action." Journal of the Academy of Nutrition and Dietetics. March. https://doi.org/10.1016/j.jand.2021.01.018. 